[When citing this chapter, refer to Behaviour 152 (2015) 407-423]

\title{
The influence of testosterone on cognitive performance in bonobos and chimpanzees
}

\author{
Victoria Wobber ${ }^{\mathrm{a}, \mathrm{b}}$ and Esther Herrmann ${ }^{\mathrm{c}, *}$ \\ ${ }^{a}$ Department of Psychology, Harvard University, William James Hall, \\ 33 Kirkland Street, Cambridge, MA 02138, USA \\ ${ }^{\mathrm{b}}$ Department of Human Evolutionary Biology, Harvard University, \\ Cambridge, MA 02138, USA \\ ${ }^{c}$ Department of Developmental and Comparative Psychology, \\ Max Planck Institute for Evolutionary Anthropology, Leipzig, Germany \\ *Corresponding author's e-mail address: eherrman@eva.mpg.de
}

Accepted 23 March 2014; published online 7 May 2014

\begin{abstract}
Levels of the steroid hormone testosterone have been found to impact diverse features of cognition from spatial memory to decision-making regarding risk, both in humans and other animals. However less is known about whether closely-related species differ in their testosterone-cognition relationships in line with pressures shaping each species' cognitive evolution. We therefore examined relationships between testosterone and cognition in two-closely related species that differ markedly in their social behaviour, cognition, and patterns of testosterone production: bonobos (Pan paniscus) and chimpanzees (Pan troglodytes). We presented individuals of both species with a battery of 16 cognitive tasks and determined whether performance on these tasks correlated with average testosterone level. We found that among male chimpanzees, high levels of testosterone correlated with higher performance in numerous tasks, including tasks assessing spatial cognition and physical cognitive abilities more broadly. Meanwhile, in male bonobos we found no correlations between testosterone and performance on the cognitive tasks, and found no correlations in females of either species. Building on prior comparative research, these results suggest that bonobos and chimpanzees differ critically in the proximate mechanisms influencing their cognitive capacities, and that in particular the role of testosterone in shaping behaviour and cognition differs dramatically between the two species.
\end{abstract}

\section{Keywords}

cognition, testosterone, chimpanzees, bonobos. 


\section{Introduction}

Understanding the proximate mechanisms that influence social behaviour and cognition can give us a deeper understanding of these traits. Steroid hormones in particular have been found to have diverse effects on behaviour and cognition, aside from their roles in facilitating reproductive and metabolic functions (Nelson, 2000; Luine, 2008; Eisenegger et al., 2011). Levels of testosterone, for example, have been found to correlate with decision-making in situations involving risk and cooperation (Burnham, 2007; Apicella et al., 2008; Stanton et al., 2011), abilities to discriminate emotion in faces (van Honk et al., 1999; Wirth \& Schultheiss, 2007; Derntl et al., 2009), and capacities pertaining to mental rotation (Hooven et al., 2004; Alexander \& Son, 2007). Meanwhile, administration of testosterone in men has been demonstrated to directly affect decision-making in cooperative interactions (Zak et al., 2009), avoid/approach responses to emotional face stimuli (Volman et al., 2011), and success in spatial memory and navigation tasks (Janowsky et al., 1994; Cherrier et al., 2001). Though research targeting the mechanisms underlying these hormone-cognition relationships is ongoing, potential means by which testosterone might influence behaviour include its effects on the amgydala and orbitofrontal cortex, specifically in reducing the links between these two areas (Mehta \& Beer, 2010; van Wingen et al., 2010; Eisenegger et al., 2011).

Many of the relationships found between testosterone and cognition in humans appear to have deep roots within our evolutionary history. Relationships between testosterone and spatial cognition have been well-documented within rodents, with testosterone enhancing performance in various types of maze tasks and even aiding in spatial memory over a temporal delay (reviewed in Leonard \& Winsauer, 2011), and more recent work suggests that testosterone mediates approach/avoidance responses to socio-emotional stimuli in male rhesus macaques, particularly in the area of increased vigilance and alertness in interaction with novel objects and response to social playbacks (Lacreuse et al., 2010, 2012). In addition, the role of testosterone in aggressive behaviour has been extensively catalogued across numerous animal taxa (Wingfield et al., 1990; Archer, 2006). Testosterone has been found to increase in the presence of reproductively active females, and facil- 
itates behaviours related to male dominance hierarchies such as displays and agonistic interactions (Muller \& Wrangham, 2004; Bartos et al., 2010). Indeed, several studies have revealed that individual and species differences in aggressive behaviour may be critically mediated by the influence of testosterone on patterns of decision-making during competitive interactions (Fuxjager \& Marler, 2010; Wobber et al., 2010).

Despite the broad research on the relationship between testosterone and cognition across taxa, few studies have directly examined whether testosterone-cognition relationships vary among closely related species in line with differing ecological pressures that those species face. Here, we propose that bonobos (Pan paniscus) and chimpanzees (Pan troglodytes) provide an ideal comparison to suit this purpose. These two species, while closely related (Won \& Hey, 2005), differ markedly in aspects of their social behaviour, cognition, and the functionality of testosterone across development and in facilitating mating effort (Muller \& Wrangham, 2004; Herrmann et al., 2010; Wobber et al., 2010, 2013; Hare et al., 2012; Surbeck et al., 2012). Bonobos show more fluid dominance hierarchies, greater aversion to risk in decision-making, and differ in their development of spatial memory capacities (Heilbronner et al., 2008; Rosati \& Hare, submitted), indicating species differences in numerous areas where testosterone has a known influence, as discussed above. These differences between bonobos and chimpanzees have been proposed to derive from fundamental shifts in feeding and social ecology between these two species, in particular with the more predictable feeding environment in the Democratic Republic of Congo facilitating heightened associations between females and corresponding reductions in male aggressive tendencies in bonobos relative to chimpanzees (Wrangham \& Pilbeam, 2001; Hare et al., 2012). We therefore hypothesized that bonobos and chimpanzees might differ significantly in their relationship between testosterone and cognition.

In this paper we discuss the results of a study examining the relationship between levels of testosterone and performance on a diverse array of cognitive tasks (the Primate Cognition Test Battery (PCTB); Herrmann et al., 2007) in bonobos and chimpanzees. We examine these relationships separately within chimpanzees and bonobos, determining the degree to which testosterone correlates with cognitive performance within each sex in each species. 


\section{Methods}

\subsection{Subjects}

We collected saliva samples from 27 chimpanzees (13 males and 14 females; 3 to 18 years of age) and 30 bonobos (19 males and 11 females; aged 5 to 22 years) who participated in the battery of cognitive tests described below and elsewhere (Herrmann et al., 2010). The chimpanzees lived at the Tchimpounga Chimpanzee Sanctuary, Republic of Congo and the bonobos at Lola ya Bonobo sanctuary, Democratic Republic of Congo. All apes came to the sanctuaries as orphans as a result of the illegal bushmeat trade and were raised together with peers, living in social groups that have access to large forest enclosures (see Wobber \& Hare (2011) for site description), except for one ape in the sample who was born on-site and mother-reared. The behavioural data discussed here have been published previously in Herrmann et al. (2010).

\subsection{Procedure}

\subsubsection{Cognitive measures: Primate Cognition Test Battery (Herrmann et al., 2007)}

Subjects were tested on the PCTB that comprised 16 physical and social cognitive different tasks (see Table 1, see original study (Herrmann et al., 2007, 2010)). The physical cognitive tasks consisted of problems concerning space, quantities and causality. The space scale comprised tasks in which the ape had to show an understanding of spatial relations by locating a reward (spatial memory), tracking a reward after invisible displacement (object permanence), tracking a reward after a rotation manipulation (rotation) or tracking a reward after visible changes in location (transposition). The quantities scale was divided into problems in which the ape had to discriminate between two quantities (relative numbers) or had to discriminate between quantities added to other quantities (addition numbers). The causality scale included tasks in which the ape had to show causal understanding either of noise produced by a hidden reward (noise), or a change in appearance produced by a hidden reward (shape). In addition, apes were presented with a task where they needed to use a stick in order to retrieve a reward which was out of reach (tool use), and a task where they needed to discriminate between a functional and a non-functional tool (tool properties). The social cognitive tasks consisted of problems concerning social learning, communication and 'theory of mind'. The social learning scale comprised a task in 
Table 1.

Primate Cognition Test Battery (PCTB) including domains, scales and tasks.

\begin{tabular}{|c|c|c|c|}
\hline Domain & Scale & Task & Description \\
\hline \multirow[t]{10}{*}{ Physical } & \multirow[t]{4}{*}{ Space } & Spatial memory & Locating a reward \\
\hline & & Object permanence & $\begin{array}{l}\text { Tracking of a reward after invisible } \\
\text { displacement }\end{array}$ \\
\hline & & Rotation & $\begin{array}{l}\text { Tracking of a reward after a rotation } \\
\text { manipulation }\end{array}$ \\
\hline & & Transposition & $\begin{array}{l}\text { Tracking of a reward after location } \\
\text { changes }\end{array}$ \\
\hline & \multirow[t]{2}{*}{ Quantities } & Relative numbers & Quantity discrimination \\
\hline & & Addition numbers & $\begin{array}{l}\text { Quantity discrimination with added } \\
\text { quantities }\end{array}$ \\
\hline & \multirow[t]{4}{*}{ Causality } & Noise & $\begin{array}{l}\text { Causal understanding of produced } \\
\text { noise by hidden rewards }\end{array}$ \\
\hline & & Shape & $\begin{array}{l}\text { Causal understanding of appearance } \\
\text { change by hidden rewards }\end{array}$ \\
\hline & & Tool use & $\begin{array}{l}\text { Using a stick in order to retrieve a } \\
\text { reward which is out of reach }\end{array}$ \\
\hline & & Tool properties & $\begin{array}{l}\text { Understanding of functional and } \\
\text { non-functional tool properties }\end{array}$ \\
\hline \multirow[t]{6}{*}{ Social } & Social learning & Social learning & $\begin{array}{l}\text { Solving a simple but not obvious } \\
\text { problem by observing a demonstrated } \\
\text { solution }\end{array}$ \\
\hline & \multirow[t]{3}{*}{ Communication } & Comprehension & $\begin{array}{l}\text { Understanding communicative cues } \\
\text { indicating a reward's hidden location }\end{array}$ \\
\hline & & Pointing cups & $\begin{array}{l}\text { Production of communicative gestures } \\
\text { in order to retrieve a hidden reward }\end{array}$ \\
\hline & & Attentional state & $\begin{array}{l}\text { Choice of communicative gestures } \\
\text { considering the attentional state of the } \\
\text { recipient }\end{array}$ \\
\hline & \multirow[t]{2}{*}{ Theory of mind } & Gaze following & $\begin{array}{l}\text { Following an actor's gaze direction to a } \\
\text { target }\end{array}$ \\
\hline & & Intentions & $\begin{array}{l}\text { Understanding what an actor intended } \\
\text { to do (unsuccessfully) }\end{array}$ \\
\hline
\end{tabular}

Table originally published in Herrmann et al. (2007).

which subjects were required to imitate another's solution to a problem (in three separate tasks of this type). The communication scale comprised one task in which the ape had to understand communicative cues indicating a reward's hidden location (comprehension) and two tasks in which subjects had to produce communicative gestures in order to retrieve a hidden reward 
(pointing cups and attentional state). The theory of mind scale was divided into a task in which the ape had to follow an experimenter's gaze to a target (gaze following) and a second task in which the subject had to infer the location of a hidden reward based on the intention-guided actions of an experimenter (intentions).

Given the known relationship between testosterone and spatial memory, we will outline the rotation task in more detail since this in fact represented a critical test for whether testosterone correlated with cognitive ability in either species. In the rotation task, apes were presented with an array of three cups sitting on a plastic platform, with a food reward hidden under one of these three cups in full view of the subject. After the food reward was hidden, the platform was rotated either 180 or 360 degrees (depending on the trial) in view of the subject. The platform was then pushed towards the subject so that he or she could identify the location of the hidden reward. If the subject did so successfully, he was rewarded; if not, he was shown the location of the food reward but not given the item. Subjects received 9 trials of this task, 3 trials with a reward hidden under the middle cup and 180 degree rotations, 3 trials with a reward hidden under either the left or the right cup and 360 degree rotations and 3 trials with a reward hidden under the either the left or right cup and 180 degree rotations, with the trials presented in a consistent order across all subjects.

\subsubsection{Hormonal measures}

To determine baseline testosterone levels of individuals participating in PCTB, we collected saliva samples from these individuals using methods outlined previously (Wobber et al., 2010, 2013) (range 1-22 samples per individual, mean 8.6 samples). Samples for an individual baseline were all collected within a 2 -month period. This two-month period fell within 1 year of when subjects participated in the PCTB. Samples were collected between 8:00 AM and 5:00 PM, with the time of day (morning versus afternoon) counterbalanced within-individual to prevent circadian rhythms from biasing the average testosterone values. Our sampling regime also avoided the early morning peak in testosterone levels upon waking seen in chimpanzees and other animals (Muller \& Lipson, 2003).

Saliva samples were collected according to procedures described elsewhere using cotton rounds and Sweet Tarts as stimulants (Wobber et al., 2010, 2013). Fifty microliters of $0.1 \%$ sodium azide solution was added 
to samples immediately after collection to prevent contamination and to allow samples to be kept at room temperature until they were returned to the laboratory (Lipson \& Ellison, 1989). The saliva samples were analysed in the Reproductive Ecology Laboratory at Harvard University. Salivary testosterone measurements were made using an ${ }^{125} \mathrm{I}$-based radioimmunoassay kit (No. 4100, Diagnostic Systems Laboratories, Webster, TX, USA) with the following modifications: standards were prepared in assay buffer and run at six concentrations from 2 to $375 \mathrm{pg} / \mathrm{ml}$. Samples were added in $100 \mu \mathrm{l}$ amounts together with $300 \mu \mathrm{l}$ of assay buffer. First antibody $(20 \mu \mathrm{l})$ and labelled steroid $(50 \mu \mathrm{l})$ were added to each tube to yield a total reaction volume of $470 \mu \mathrm{l}$ per tube. After overnight incubation at $4^{\circ} \mathrm{C}, 500 \mu \mathrm{l}$ of second antibody was added to each reaction tube. Reaction tubes were subsequently centrifuged for $45 \mathrm{~min}$; after aspiration of the supernatant, tubes were counted in a gamma counter for $2 \mathrm{~min}$. In pilot assays, the ape testosterone values using the standard aliquot for human assays $(200 \mu \mathrm{l})$ were too high to be readable in the assay range. Thus, we used only $100 \mu \mathrm{l}$ of the chimpanzee and bonobo saliva for the T assays, with the same standard curve as employed in the human testosterone radioimmunoassay protocol. Validation of this sample collection and analysis procedure is discussed in Wobber et al. (2013).

\subsection{Analyses}

We first computed an average testosterone value for each individual. Then, we log-transformed these average testosterone values to normalize our data and enable the use of parametric statistics. Below, we first provide descriptive statistics on the testosterone values, to confirm that there was comparable variance in between sexes in each species within this subsample taken from a larger project examining testosterone production throughout development in bonobos and chimpanzees (Wobber et al., 2013). Similarly, we discuss overall variance in the cognitive measures. Second, we discuss the results of Pearson correlations measuring the relationship between an individual's average testosterone level and his or her performance on (1) each broad division of the test battery (physical versus social) and (2) each of the six cognitive scales described above: space, quantities, tools and causality, social learning, communication, and 'theory of mind'. For the scales where we found a significant relationship between testosterone and performance, we analysed task-by-task correlations with testosterone to determine which 
tasks might best predict the overall relationship. Finally, we performed partial correlations to ensure that age did not mediate any relationships between testosterone and cognitive performance, given that our previous findings have established that testosterone and cognitive task performance increase more markedly with age in chimpanzees than in bonobos (Wobber et al., 2013, unpublished data). Given the known sex differences in testosterone levels in mammals, we performed these analyses separately for males and females within each species.

\section{Results}

Descriptive statistics showed that variability in testosterone levels was comparable between males of the two species, as was variation in performance across the two main cognitive divisions (Table 2). Variance in testosterone levels was higher among female chimpanzees due to one female with extremely high testosterone levels. Finally, variance in performance on the cognitive tasks was comparable between the two species, with the only difference that female bonobos showed slightly more variance in cognitive performance than female chimpanzees (meaning that if anything, we would be more likely to find a relationship between testosterone and cognitive performance in female bonobos given their greater variance on these measures) (Table 2). Average testosterone levels in each species and sex were as follows: male chimpanzees $0.27 \mathrm{ng} / \mathrm{ml}$, male bonobos $0.22 \mathrm{ng} / \mathrm{ml}$, female chimpanzees $0.18 \mathrm{ng} / \mathrm{ml}$, female bonobos $0.17 \mathrm{ng} / \mathrm{ml}$. Note that we performed statistical analyses with log-transformed testosterone values, with the rationale discussed in our 'Analyses' section above.

Examining the results of Pearson correlations between log average testosterone and performance on the cognitive measures, within male chimpanzees we found several significant relationships between log average testosterone values and performance on the cognitive tasks. Males with higher testosterone on average performed more skilfully in the physical cognition division of tasks ( $r=0.795, p=0.001, N=13)$, though they did not perform more skilfully within the social cognition division (Figure 1, Table 3). Looking at the cognitive scale level, male chimpanzees with higher testosterone levels performed more skilfully in space $(r=0.620, p=0.024, N=13)$ as well as causality $(r=0.588, p=0.035, N=13)$. Within these two scales, taskby-task analysis only revealed one task-level correlation between performance and testosterone level, with higher levels of testosterone correlating 


\section{Table 2.}

Descriptive statistics for testosterone levels and performance on the two major cognitive divisions (physical versus social cognition) and 6 cognitive scales for each species and sex.

\begin{tabular}{|c|c|c|c|c|c|c|c|c|}
\hline & \multicolumn{4}{|c|}{ Males } & \multicolumn{4}{|c|}{ Females } \\
\hline & \multicolumn{2}{|c|}{ Chimpanzees } & \multicolumn{2}{|c|}{ Bonobos } & \multicolumn{2}{|c|}{ Chimpanzees } & \multicolumn{2}{|c|}{ Bonobos } \\
\hline & SE & Range & SE & Range & SE & Range & SE & Range \\
\hline Log testosterone & 0.07 & 0.76 & 0.07 & 0.75 & 0.09 & 1.11 & 0.05 & 0.48 \\
\hline Physical cognition & 0.02 & 0.22 & 0.01 & 0.24 & 0.01 & 0.16 & 0.02 & 0.24 \\
\hline Social cognition & 0.02 & 0.26 & 0.02 & 0.32 & 0.02 & 0.24 & 0.03 & 0.35 \\
\hline Space & 0.03 & 0.39 & 0.03 & 0.53 & 0.02 & 0.25 & 0.03 & 0.36 \\
\hline Quantities & 0.03 & 0.36 & 0.03 & 0.42 & 0.03 & 0.30 & 0.03 & 0.34 \\
\hline Causality & 0.03 & 0.37 & 0.02 & 0.32 & 0.04 & 0.42 & 0.04 & 0.36 \\
\hline Social learning & 0.04 & 0.33 & 0.03 & 0.33 & 0.03 & 0.25 & 0.05 & 0.33 \\
\hline Communication & 0.04 & 0.41 & 0.03 & 0.52 & 0.03 & 0.42 & 0.06 & 0.73 \\
\hline Theory of mind & 0.03 & 0.39 & 0.03 & 0.56 & 0.03 & 0.47 & 0.03 & 0.39 \\
\hline
\end{tabular}

with more skilful performance on the rotation task $(r=0.601, p=0.030$, $N=13$ ). Meanwhile, there was also one scale where higher levels of testosterone were correlated with poorer performance in male chimpanzees: the theory of mind scale ( $r=-0.598, p=0.031, N=13$ ), and within that scale on the gaze-following task $(r=-0.596, p=0.030, N=13)$ (Table 3$)$.

We next examined the potential co-variation of age with these relationships between testosterone and performance in male chimpanzees. We performed partial correlations between log average testosterone and performance on the scales/tasks where there was a significant relationship in the Pearson correlations and controlled for the effect of age as a covariate. In these partial correlations, only the positive relationships between testosterone and physical cognition (partial correlation with age, $r=0.696$, $p=0.012, \mathrm{df}=10)$ and between testosterone and the rotation task (partial correlation with age, $r=0.616, p=0.033$, $\mathrm{df}=10$ ) remained significant. These correlations indicate that testosterone had an independent effect on physical cognition and rotation in particular, even when taking into account any effect that learning or heightened experience in interacting with the world might have conferred in terms of advantages on these tests for older individuals. 
(A)

\section{Physical cognition}

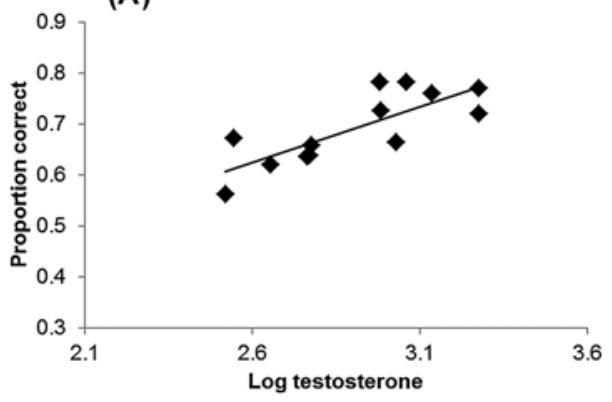

(B)

\section{Physical cognition}

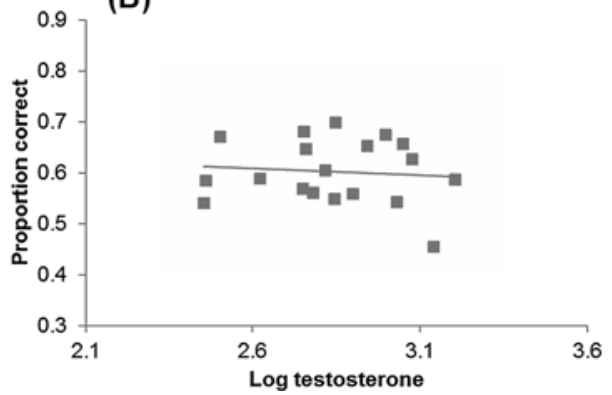

Figure 1. Regression of average log testosterone levels and average performance across 10 physical cognition tasks in (A) chimpanzee males and (B) bonobo males. Each point represents one individual (either a chimpanzee, as depicted by the black diamond, or a bonobo, as depicted by the grey squares), and that individual's average testosterone level (log-transformed) as well as their average proportion correct across the physical cognition tasks. Regression lines denoting for the relationship between testosterone and proportion correct within the physical cognition division are shown for each species.

Turning to male bonobos, we found no significant relationships between log average testosterone and performance on any divisions or scales of the cognitive test battery, despite our larger sample of male bonobos $(N=19)$ relative to male chimpanzees $(N=13)$. Similarly, no significant relationships emerged even when controlling for the effect of age with partial correlations. These findings suggest a stronger role of testosterone in association with performance on cognitive tasks in chimpanzees relative to bonobos, despite there being similar levels of variance in task performance and testosterone level in males within our sample (Table 2).

Among females, there was only one significant relationship between testosterone and performance in either species. Female chimpanzees showed 


\section{Table 3.}

Relationship between testosterone and cognitive performance in male chimpanzees $(N=13)$, male bonobos $(N=19)$, female chimpanzees $(N=14)$ and female bonobos $(N=11)$.

\begin{tabular}{|c|c|c|c|c|c|c|c|c|}
\hline & \multicolumn{4}{|c|}{ Males } & \multicolumn{4}{|c|}{ Females } \\
\hline & \multicolumn{2}{|c|}{ Chimpanzees } & \multicolumn{2}{|c|}{ Bonobos } & \multicolumn{2}{|c|}{ Chimpanzees } & \multicolumn{2}{|c|}{ Bonobos } \\
\hline & $r$ & $p$ & $r$ & $p$ & $r$ & $p$ & $r$ & $p$ \\
\hline Physical cognition & 0.795 & $0.001^{*}$ & -0.095 & 0.698 & -0.356 & 0.211 & 0.098 & 0.774 \\
\hline Social cognition & -0.140 & 0.648 & 0.092 & 0.708 & 0.363 & 0.202 & -0.503 & 0.115 \\
\hline Space & 0.620 & 0.024 & -0.162 & 0.509 & -0.448 & 0.108 & -0.357 & 0.282 \\
\hline Quantities & 0.199 & 0.516 & -0.045 & 0.853 & 0.016 & 0.957 & 0.192 & 0.571 \\
\hline Causality & 0.588 & 0.035 & 0.112 & 0.649 & -0.168 & 0.567 & 0.282 & 0.400 \\
\hline Social learning & -0.055 & 0.865 & 0.196 & 0.422 & 0.065 & 0.848 & -0.311 & 0.352 \\
\hline Communication & -0.200 & 0.512 & 0.318 & 0.184 & 0.577 & $0.031^{*}$ & -0.263 & 0.434 \\
\hline Theory of mind & -0.598 & 0.031 & -0.343 & 0.151 & -0.166 & 0.570 & -0.496 & 0.121 \\
\hline
\end{tabular}

Pearson correlation values $(r)$ are indicated for the relationships between log average testosterone and the overall cognitive task divisions (physical versus social cognition), as well as each of the 6 cognitive scales, along with their corresponding $p$-values. Italicised values indicate a positive correlation.

* Results of a partial correlation controlling for the effect of age were significant.

a positive correlation between log average testosterone and performance in the communication scale $(r=0.577, p=0.031, N=14)$ (Table 3), which was likely influenced by a strong relationship between testosterone and performance on the attentional state task $(r=0.665, p=0.009, N=14)$. These relationships remained significant even after controlling for age (partial correlations, communication scale: $r=0.569, p=0.042, \mathrm{df}=11$, attentional state task: $r=0.755, p=0.003, \mathrm{df}=11$ ). Meanwhile, there were no significant relationships between testosterone and performance on any division or scale of the test battery in female bonobos, even after partialling out the effects of age - though this was the group in our analysis with the smallest sample size.

\section{Discussion}

Our results reveal significant differences between bonobos and chimpanzees in the relationship between testosterone and performance on cognitive tasks, particularly among males. Among chimpanzee males, higher levels of testosterone correlated with higher levels of performance in the physical cognition 
division on the whole, as well as with the space and causality scales. In addition, among male chimpanzees, higher levels of testosterone correlated with lower levels of theory of mind performance. Meanwhile among bonobo males there were no relationships between testosterone and performance on the cognitive tasks, despite having a larger sample size of male bonobos relative to male chimpanzees and the two groups showing comparable levels of variance in these measures. Finally, among female chimpanzees there was a positive relationship between testosterone level and performance on the communication scale, largely driven by higher levels of testosterone correlating with more skilful performance in the attentional state task. Among female bonobos there were no significant relationships between testosterone and cognitive performance.

These findings indicate that in a number of areas where testosterone has been implicated in human and rodent cognition including spatial cognition (Williams \& Meck, 1991; Silverman et al., 1999; Hooven et al., 2004; Leonard \& Winsauer, 2011), chimpanzees also show a significant role of testosterone level in accounting for individual variance. This relationship was particularly apparent in the rotation task, where individuals needed to track a reward after the reward locations were rotated geometrically. Females of both species showed fewer significant relationships between testosterone and performance - the association between testosterone and behaviour/cognition has been less studied in females than in males, with greater inconclusiveness of findings (Bateup et al., 2002), though see Beehner et al. (2005), prompting further inquiry in this area.

Importantly, male chimpanzees and bonobos differed in their relationship between testosterone and performance on the cognitive tasks administered here. These results align with the finding that testosterone plays a significant role in numerous aspects of chimpanzee social life — with males differing in baseline testosterone level in correlation with dominance rank (Muehlenbein et al., 2004; Muller \& Wrangham, 2004), showing changes in testosterone levels when females are most fecund (Muller \& Wrangham, 2004), and showing more responsiveness in levels of testosterone surrounding competitive interactions (Wobber et al., 2010; Sobolewski et al., 2012). In contrast, in bonobos these behaviours have been found to correlate less well with levels of testosterone, with male bonobos showing a weaker association between testosterone and rank (Marshall \& Hohmann, 2005; Surbeck et al., 2012), little change in testosterone level depending on female fecundity (Surbeck et 
al., 2012), minimal change in testosterone level surrounding varying types of competitive interaction (Wobber et al., 2010), and even lesser change in testosterone level throughout development relative to chimpanzees (Wobber et al., 2013). On the whole then, these findings suggest that testosterone may play a greater role in chimpanzee social behaviour and cognition than found among bonobos, suggesting striking differences in the proximate mechanisms underlying behaviour and cognition in these two species. In line with the broader cognitive differences between chimpanzees and bonobos then (Herrmann et al., 2010), the associations found here between testosterone and cognition in chimpanzees, but not bonobos, suggests one potential mechanism by which the behavioural and cognitive variation between these two ape taxa might be maintained.

Nonetheless, our results have demonstrated a correlative, rather than a causal relationship; hence, it is possible that testosterone's effect on male chimpanzees' performance on specific cognitive tasks is mediated via changes in visual attention or motivation to participate in these tasks. However, if that was the case, it would not explain why testosterone correlated only with specific tasks and scales rather than performance overall. Furthermore, we were also able to rule out age as a potential covariate driving the relationships between testosterone and at least some of the cognitive scales in male chimpanzees. It is also possible that greater ability in certain areas, such as tracking potential feeding trees in space, in fact enables males to attain higher rank and therefore to sustain a higher level of testosterone. It is therefore possible that heightened cognitive abilities drive attainment of rank and increases in testosterone levels, rather than testosterone levels facilitating differing cognitive acuity, as we have postulated here. Further inquiry experimentally inducing testosterone increases and measuring their potential effects on cognitive performance in male apes can tease apart elements of causality in the testosterone-cognition relationship.

This study provides greater insight into the role of testosterone of these two closely-related species, but additional research is necessary (1) to clarify the mechanisms by which testosterone might influence cognition, including manipulations of testosterone level to determine whether this might directly impact performance (Zak et al., 2009; Volman et al., 2011) and (2) to identify why relationships between testosterone and cognition might be less significant among bonobos relative to chimpanzees, in line with the differences in social behaviour, cognition and endocrinology between the two species (Her- 
rmann et al., 2010; Hare et al., 2012; Wobber et al., 2013). Moreover, work with additional steroid hormones such as oestradiol and androstenedione can clarify the specific mechanisms by which hormone levels mediate individual differences in cognition across species, including humans (Azurmendi et al., 2005; Luine, 2008).

\section{Acknowledgements}

We are grateful to both sanctuaries for hosting our research. We are thankful to L. Pharoah, R. Atencia, K. Brown and the Jane Goodall Institute USA and staff of Tchimpounga Sanctuary and Claudine Andre, Dominique Morel, Crispin Kamate Mahamba, and Pierrot Mbonzo and the staff Lola ya Bonobo Sanctuary for their enthusiasm and support. In particular, we appreciate the hard work of the animal caregivers: J. Maboto, B. Moumbaka, A. Sitou, M. Makaya, B. Bissafi, C. Ngoma, W. Bouity, J.A. Tchikaya, L. Bibimbou, A. Makosso, C. Boukindi, G. Nzaba, B. Ngoma, J.C. Nzumbi, S. Mokando, C. Paluku, A. Kisungu, P. Kunaka, N. Luvualu and K. Manzambi. We also thank the Ministry of Environment in the Democratic Republic of Congo and the Congolese Ministere de la Recherche Scientifique et de l'Innovation Technique in the Republic of Congo. Finally, we thank Peter Ellison and Susan Lipson for their help in performing the endocrine analyses. This research was approved by the Institutional Care and Use Committees at Harvard University, and complied with the laws of the countries in which it was performed. This work was supported in part by an L.S.B. Leakey Foundation Grant, National Science Foundation BCS-0851291 (DDIG), and a WennerGren Foundation Grant to V.W. as well as NSF grants BCS-08-27552 and BCS-25172 to B.H.

\section{References}

Alexander, G.M. \& Son, T. (2007). Androgens and eye movements in women and men during a test of mental rotation ability. - Horm. Behav. 52: 197-204.

Apicella, C.L., Dreber, A., Campbell, B., Gray, P.B., Hoffman, M. \& Little, A.C. (2008). Testosterone and financial risk preferences. — Evol. Hum. Behav. 29: 384-390.

Archer, J. (2006). Testosterone and human aggression: an evaluation of the challenge hypothesis. - Neurosci. Biobehav. Rev. 30: 319-345.

Azurmendi, A., Braza, F., Sorozabal, A., Garcia, A., Braza, P., Carreras, M., Munoz, J., Cardas, J. \& Sanchez-Martin, J. (2005). Cognitive abilities, androgen levels, and body mass index in 5-year-old children. - Horm. Behav. 49: 187-195. 
Bartos, L., Schams, D., Bubenik, G.A., Kotrba, R. \& Tomanek, M. (2010). Relationship between rank and plasma testosterone and cortisol in red deer males (Cervus elaphus). - Physiol. Behav. 101: 628-634.

Bateup, H., Booth, A., Shirtcliff, E. \& Granger, D. (2002). Testosterone, cortisol, and women's competition. - Evol. Hum. Behav. 23: 181-192.

Beehner, J., Phillips-Conroy, J. \& Whitten, P. (2005). Female testosterone, dominance rank, and aggression in an Ethiopian population of hybrid baboons. - Am. J. Primatol. 67: 101-119.

Burnham, T.C. (2007). High-testosterone men reject low ultimatum game offers. - Proc. Roy. Soc. Lond. B: Biol. Sci. 274: 2327-2330.

Cherrier, M.M., Asthana, S., Plymate, S., Baker, L., Matsumoto, A.M., Peskind, E., Raskind, M.A., Brodkin, K., Bremner, W., Petrova, A., LaTendresse, S. \& Craft, S. (2001). Testosterone supplementation improves spatial and verbal memory in healthy older men. Neurology 57: 80-88.

Derntl, B., Windischberger, C., Robinson, S., Kryspin-Exner, I., Gur, R.C., Moser, E. \& Habel, U. (2009). Amygdala activity to fear and anger in healthy young males is associated with testosterone. - Psychoneuroendocrinology 34: 687-693.

Eisenegger, C., Haushofer, J. \& Fehr, E. (2011). The role of testosterone in social interaction. — Trends Cogn. Sci. 15: 263-271.

Fuxjager, M. \& Marler, C. (2010). How and why the winner effect forms: influences of contest environment and species differences. - Behav. Ecol. 21: 37-45.

Hare, B., Wobber, V. \& Wrangham, R. (2012). The self-domestication hypothesis: bonobo psychology evolved due to selection against aggression. - Anim. Behav. 83: 573-585.

Heilbronner, S., Rosati, A., Stevens, J., Hare, B. \& Hauser, M. (2008). A fruit in the hand or two in the bush? Divergent risk preferences in chimpanzees and bonobos. - Biol. Lett. 4: 246-249.

Herrmann, E., Call, J., Hernandez-Lloreda, M., Hare, B. \& Tomasello, M. (2007). Humans have evolved specialized skills of social cognition: the cultural intelligence hypothesis. Science 317: 1360-1366.

Herrmann, E., Hare, B., Call, J. \& Tomasello, M. (2010). Differences in the cognitive skills of bonobos and chimpanzees. - PLOS One 5: e12438.

Hooven, C., Chabris, C., Ellison, P. \& Kosslyn, S. (2004). The relationship of male testosterone to components of mental rotation. - Neuropsychologia 42: 782-790.

Janowsky, J.S., Oviatt, S.K. \& Orwoll, E.S. (1994). Testosterone influences spatial cognition in older men. - Behav. Neurosci. 108: 325-332.

Lacreuse, A., King, H.M., Kurdziel, L.B., Partan, S.R., Caldwell, K.M., Chiavetta, M.R., Millette, M.M., Meyer, J.S. \& Grow, D.R. (2010). Testosterone may increase selective attention to threat in young male macaques. - Horm. Behav. 58: 854-863.

Lacreuse, A., Gore, H.E., Chang, J. \& Kaplan, E.R. (2012). Short-term testosterone manipulations modulate visual recognition memory and some aspects of emotional reactivity in male rhesus monkeys. — Physiol. Behav. 106: 229-237.

Leonard, S.T. \& Winsauer, P.J. (2011). The effects of gonadal hormones on learning and memory in male mammals: a review. - Curr. Zool. 57: 543-558. 
Luine, V.N. (2008). Sex steroids and cognitive function. - J. Neuroendocrinol. 20: 866-872. Marshall, A. \& Hohmann, G. (2005). Urinary testosterone levels of wild male bonobos (Pan paniscus) in the Lomako forest, Democratic Republic of Congo. - Am. J. Primatol. 65: 87-92.

Mehta, P.H. \& Beer, J. (2010). Neural mechanisms of the testosterone-aggression relation: the role of orbitofrontal cortex. - J. Cogn. Neurosci. 22: 2357-2368.

Muehlenbein, M., Watts, D. \& Whitten, P. (2004). Dominance rank and fecal testosterone levels in adult male chimpanzees (Pan troglodytes schweinfurthii) at Ngogo, Kibale National Park, Uganda. - Am. J. Primatol. 64: 71-82.

Muller, M. \& Lipson, S. (2003). Diurnal patterns of urinary steroid excretion in wild chimpanzees. - Am. J. Primatol. 60: 161-166.

Muller, M. \& Wrangham, R. (2004). Dominance, aggression and testosterone in wild chimpanzees: a test of the 'challenge hypothesis'. - Anim. Behav. 67: 113-123.

Nelson, R. (2000). An introduction to behavioral endocrinology. - Sinauer Associates, Sunderland, MA.

Silverman, I., Kastuk, D., Choi, J. \& Phillips, K. (1999). Testosterone levels and spatial ability in men. - Psychoneuroendocrinology 24: 813-822.

Sobolewski, M., Brown, J. \& Mitani, J.C. (2012). Territoriality, tolerance and testosterone in wild chimpanzees. - Anim. Behav. 84: 1469-1474.

Stanton, S.J., Liening, S.H. \& Schultheiss, O.C. (2011). Testosterone is positively associated with risk taking in the Iowa Gambling Task. - Horm. Behav. 59: 252-256.

Surbeck, M., Deschner, T., Schubert, G., Weltring, A. \& Hohmann, G. (2012). Mate competition, testosterone and intersexual relationships in bonobos, Pan paniscus. - Anim. Behav. 83: 659-669.

van Honk, J., Tuiten, A., Verbaten, R., van den Hout, M., Koppeschaar, H., Thijssen, J. \& de Haan, E. (1999). Correlations among salivary testosterone, mood, and selective attention to threat in humans. - Horm. Behav. 36: 17-24.

van Wingen, G., Mattern, C., Verkes, R.J., Buitelaar, J. \& Fernandez, G. (2010). Testosterone reduces amygdala-orbitofrontal cortex coupling. - Psychoneuroendocrinology 35: 105113.

Volman, I., Toni, I., Verhagen, L. \& Roelofs, K. (2011). Endogenous testosterone modulates prefrontal-amygdala connectivity during social emotional behavior. - Cerebr. Cort. 21: 2282-2290.

Williams, C.L. \& Meck, W.H. (1991). The organizational effects of gonadal-steroids on sexually dimorphic spatial ability. - Psychoneuroendocrinology 16: 155-176.

Wingfield, J., Hegner, R., Dufty, R. \& Ball, G. (1990). The "challenge hypothesis": theoretical implications for patterns of testosterone secretion, mating systems, and breeding strategies. - Am. Nat. 136: 829-846.

Wirth, M. \& Schultheiss, O. (2007). Basal testosterone moderates responses to anger faces in humans. - Physiol. Behav. 90: 496-505.

Wobber, V. \& Hare, B. (2011). Psychological health of orphan bonobos and chimpanzees in African sanctuaries. - PLOS One 6: e17147. 
Wobber, V., Hare, B., Maboto, J., Lipson, S., Wrangham, R. \& Ellison, P. (2010). Differential changes in steroid hormones before competition in bonobos and chimpanzees. - Proc. Natl. Acad. Sci. USA 107: 12457-12462.

Wobber, V., Lipson, S., Hare, B., Wrangham, R. \& Ellison, P. (2013). Different ontogenetic patterns of testoterone production reflect divergent male reproductive strategies in chimpanzees and bonobos. — Physiol. Behav. 116-117: 44-53.

Won, Y. \& Hey, J. (2005). Divergence population genetics of chimpanzees. - Mol. Biol. Evol. 22: 297-307.

Wrangham, R. \& Pilbeam, D. (2001). African apes as time machines. - In: All apes great and small (Galdikas, B., Briggs, N., Sheeran, L., Shapiro, G. \& Goodall, J., eds). Kluwer Academic/Plenum Publishers, New York, NY, p. 5-18.

Zak, P.J., Kurzban, R., Ahmadi, S., Swerdloff, R.S., Park, J., Efremidze, L., Redwine, K., Morgan, K. \& Matzner, W. (2009). Testosterone administration decreases generosity in the ultimatum game. - PLOS One 4: 7. 